\title{
Teaching literature in the French language and literature departments in Greece: research methodologies and results of a quantitative and qualitative survey
}

\section{[L'enseignement de la littérature dans les départements de langue et de littérature françaises en Grèce : méthodologies de recherche et résultats d'une enquête quantitative et qualitative]}

\author{
Angeliki Kordoni
}

DOI: 10.18355/XL.2018.11.01XL.23

\begin{abstract}
The purpose of this study is to present the methodology of research followed during my Doctoral thesis defended in Sorbonne Nouvelle-Paris 3 University in April 2015. This doctoral thesis in Teaching Languages and Cultures aimed to examine French Literature teaching in the Greek university system in order to establish its main features. The two departments of « French Language and Literature » in Athens and in Thessaloniki constituted our field of observation. Relying on questionnaires and interviews with students and professors, we attempted to detect the representations, the nature of choices made in relation to texts and commonly adopted approaches to literature instruction. This thesis cross-references students' expectations and conceptions with those stipulated in handbooks and proposed by professors.
\end{abstract}

Key words: teaching of literature, French as a Foreign Language, research methodologies, methodological triangulation, quantitative research, qualitative research

\section{Résumé}

Cet article a pour objectif de présenter la méthodologie de recherche suivie lors de notre thèse doctorale soutenue à l'Université Sorbonne Nouvelle Paris 3 en avril 2015. Notre thèse en Didactique des langues et des cultures avait pour objectif d'étudier l'enseignement de la littérature française dans le système universitaire grec afin d'en dégager ses principales caractéristiques. Les deux départements de «Langue et de Littérature françaises » à Athènes et à Thessalonique ont constitué le terrain de notre observation. En s'appuyant sur des questionnaires soumis aux étudiants et professeurs et sur des entretiens menés avec nombre d'entre eux, elle tente de déceler les représentations, la nature des choix effectués en matière de textes ainsi que les approches adoptées pour l'enseignement de la littérature. Cette thèse croise les attentes et les conceptions des étudiants avec celles induites par les manuels mais aussi avec celles proposées par les professeurs universitaires.

Mots-clés : enseignement de la littérature, Français Langue Étrangère, méthodologies de recherche, triangulation méthodologique, enquête quantitative, enquête qualitative

\section{Introduction et hypothèses de recherche}

Cet article vise à présenter la méthodologie de la recherche adoptée lors de notre thèse de doctorat, soutenue à l'Université Sorbonne Nouvelle Paris 3 en avril 2015. Il se focalise sur la description des différentes étapes méthodologiques suivies lors de notre recherche. La première partie de ce travail consiste à la présentation du sujet, du terrain, de nos questions de recherche et de nos hypothèses. La deuxième partie est consacrée à la description de deux types de méthodes que nous avons utilisés ; le questionnaire et l'entretien. Nous expliquerons la structure du questionnaire, le guide et le déroulement des entretiens, le recueil des données ainsi que leur traitement. 
Néanmoins, le volume important de nos résultats rend impossible leur analyse dans un article d'une longueur limitée et c'est pour cela que nous présenterons qu'un bref aperçu de ceux-ci.

Cette étude est née de la volonté de questionner la place de la littérature française dans les cursus universitaires grecs, les images, les représentations, les approches et les contenus de cet enseignement. Nous avons donc déterminé comme terrains d'étude les deux Départements de "Langue et de Littérature françaises » de l'Université Nationale Capodistrienne d'Athènes et de l'Université Aristote de Thessalonique. Les deux Départements, qui sont d'ailleurs les seuls chargés de la formation des futurs enseignants de FLE en Grèce, visent surtout au perfectionnement de la langue et à l'étude de la littérature française. Leurs cursus d'études offrent aux étudiants la possibilité de suivre des formations différentes, à savoir, la littérature et la civilisation françaises, la linguistique, la didactique et la traduction.

Notre familiarisation avec le terrain s'explique par le fait d'avoir été étudiante au niveau «Licence » et "Master» à l'Université Aristote de Thessalonique. Tout au long de nos six années d'études, nos expériences personnelles nous ont permis de construire une certaine image de la littérature et de son enseignement. Les situations, les observations ainsi que les difficultés auxquelles nous avons été confrontées ont stimulé notre intérêt et nous ont amenées à nous poser des questions. En outre, une autre motivation, cette fois extrinsèque, nous a conduites à réaliser cette recherche : la nouvelle réforme des cursus d'études qui a eu lieu en 2009-2010 au sein de deux Départements. Les enseignants ont procédé, pour la première fois, à une réforme et à une réorganisation de la maquette des enseignements offerts. En examinant les maquettes, nous avons relevé que les cours littéraires ne suivaient plus l'ordre chronologique proposé par les manuels de Lagarde et Michard utilisés pendant environ 30 années consécutives. Plusieurs nouveaux modules sont apparus dans les cursus de deux départements et l'initiation des étudiants à la littérature française semblait se réaliser de manière plus douce.

Ces constatations nous ont amenées à nous interroger sur différents sujets " problèmes » concernant: la place et le statut de la littérature dans l'ancien et le nouveau cursus d'études, les manuels utilisés, les textes et les auteurs abordés, les méthodologies adoptées, la place de l'étudiant dans le processus d'enseignement, les représentations et les images suscitées ainsi que les propositions éventuelles susceptibles de contribuer à une rénovation de l'enseignement de la littérature.

Les premiers mois de notre recherche ont été consacrés à la constitution de notre corpus bibliographique. Cette étape a alimenté notre réflexion et nous a aidé à redéfinir les questionnements de notre recherche, à formuler notre problématique et à élaborer nos hypothèses. Notre recherche a d'abord commencé avec une hypothèse générale concernant l'enseignement de la littérature. Nous avons supposé que les modifications des cursus ont été surtout réalisées car les enseignants ont constaté le désintérêt des étudiants pour les cours littéraires. Les amphithéâtres vides les ont conduits à réaliser que les cours littéraires ne correspondaient pas au niveau linguistique des étudiants et ne satisfaisaient pas leurs attentes.

Progressivement, nous avons commencé à construire des hypothèses plus concrètes et plus testables. Nous avons émis comme première hypothèse que l'absence de la littérature contemporaine et des genres familiers aux pratiques des étudiants est une des raisons de ce désintérêt pour les cours de littérature. L'enseignement du «Panthéon littéraire hexagonal » pourrait conférer un caractère sacré et une image élitiste. Cette vision pourrait constituer un frein à l'apprentissage, bloquer la motivation des étudiants et conduire à une résistance psychologique. Par la suite, nous avons supposé que la majorité des étudiants n'appréciaient pas les cours littéraires à cause de leur caractère trop théorique qui encourageait leur passivité. Les cours littéraires proposés par les deux départements exigeaient surtout la mémorisation et l'apprentissage par cœur de l'analyse des extraits, des éléments biographiques des 
auteurs et des mouvements littéraires. Or, le développement de l'esprit de partage et d'échange entre les étudiants mais aussi entre les étudiants et les enseignants pourrait être capable d'améliorer l'image de la littérature. Selon notre troisième hypothèse, l'intégration aux cours littéraires des Technologies de l'Information et de la Communication pour l'Éducation (TICE) serait capable de renforcer la motivation des étudiants et de favoriser leur apprentissage. Nous avons donc supposé que l'accès aux TICE augmenterait l'intérêt des étudiants pour le cours, favoriserait leur engagement face à l'apprentissage et améliorerait la compréhension et l'approfondissement des cours.

\section{Les types de recherche adoptés}

La formulation de nos hypothèses et la consultation de différents ouvrages sur la méthodologie de recherche nous ont permis de déterminer le type et les méthodes de notre enquête ainsi que les processus et les techniques qui en découlent. Ces lectures nous ont conduits à deux approches de recherche : l'approche quantitative et l'approche qualitative. La première vise en général à recueillir des données observables, à contrôler et à prédire. Elle aboutit à des données chiffrées, à des tableaux, à des graphiques et à des analyses de corrélation (Poisson, 1983 : 370). La deuxième vise à comprendre et à décrire un phénomène et elle fournit des données de contenu. Les chercheurs de l'approche qualitative tentent de saisir la réalité telle que la vivent les sujets et ils essaient de pénétrer à l'intérieur de l'univers observé (Poisson, 1983 : 371). En effet, les études quantitatives s'attachent à répondre au " combien » tandis que les études qualitatives au «comment» et au «pourquoi ».

Dans notre cas, les deux approches nous ont permis de récolter plus d'informations et elles ont été complémentaires l'une à l'autre. Cette démarche méthodologique « mixte » nous a permis de combiner les données et les méthodes d'une façon cohérente, afin d'enrichir les résultats et les observations de notre recherche (Karsenti, $2006: 4$ ) et d'avoir une vision plus globale de la réalité. Afin d'examiner les formes que prend la littérature dans les deux départements, nous avons décidé de croiser non seulement les types d'enquête mais aussi les différents types de documents et des ressources. Les chercheurs expliquent que la diversification des angles d'observation, par le biais de la triangulation, peut nous permettre d'augmenter la validité des analyses et d'améliorer la précision de la description (Habhab, 2006 : 60-63).

Nous nous sommes inspirées du concept de triangulation attribué aux travaux de Norman K. Denzin (1970). Tout d'abord, nous avons examiné les maquettes de formation, les cours littéraires offerts ainsi que leurs descriptifs afin de déceler les finalités assignées et les modalités de leur approche. Parallèlement, nous avons étudié les manuels et les textes enseignés, qui sont, le plus souvent, vecteurs d'une certaine conception de ce qu'est la littérature. Puis, nous avons analysé les réponses des questionnaires proposés ainsi que les discours des entretiens avec les enseignants et les étudiants portant sur les pratiques d'enseignement et d'apprentissage. Enfin, l'étude de notre objet à l'aide de différentes théories ainsi que la transdisciplinarité de notre thèse nous ont permis d'éclairer et d'approfondir notre réflexion. 


\begin{tabular}{|l|l|}
\hline $\begin{array}{l}\text { a. Triangulation de sources différentes } \\
\text { dans le temps, l'espace, les personnes }\end{array}$ & $\begin{array}{l}\text { - corpus, manuels, textes utilisés dans le } \\
\text { temps. } \\
\text { - comparaison des corpus dans l'espace : } \\
\text { Athènes et Thessaloniki } \\
\end{array}$ \\
\hline b. Triangulation de méthodes différentes & comparaison de l'utilisation de ces \\
& - le questionnaire \\
& - l'entretien \\
& - l'observation \\
\hline c. Triangulation théorique & - la didactique de la littérature \\
& - la didactique du FLE \\
& - la théorie littéraire \\
& - la sociologie de la lecture \\
\hline
\end{tabular}

\section{L'enquête quantitative et certains résultats}

Notre échantillon était constitué de 126 étudiants, dont 112 femmes et 14 hommes de l'Université Nationale Capodistrienne et de l'Université Aristote. La première série de questions (A) comprenait des questions relatives à l'échantillonnage par quotas et nous informait sur l'identité des participants (prénom, âge, sexe, langue maternelle), leur parcours universitaire et leur niveau linguistique avant leur inscription à l'Université (certificats de langues obtenus). Par la suite, nous nous sommes intéressés à leurs pratiques de lecture. Cette deuxième série $(\mathrm{B})$ de trois questions intégrait les lectures littéraires en langue française ainsi que les lectures extra-scolaires pour le plaisir. La troisième série $(\mathrm{C})$ de questions avait plusieurs objectifs : connaître le contenu des cours littéraires et les méthodes d'enseignement, examiner la satisfaction des étudiants et savoir s'ils les considéraient indispensables pour leurs cursus (voir le questionnaire an Annexe).

Au sein de notre questionnaire, nous avons utilisé plusieurs types de questions, selon nos objectifs. La plupart d'entre elles étaient des questions fermées dichotomiques, multichotomiques ainsi que des questions avec classement hiérarchique où les étudiants devaient organiser les réponses en octroyant la première place à la réponse la plus importante et la dernière place à la moins importante. Les questions avec échelle de fréquence nous ont permis de comprendre ce qui se passait dans les classes des universités et nous ont donné la possibilité d'avoir une vision globale du déroulement du cours et des activités mises en place. Finalement, la dernière partie de notre questionnaire comprenait des questions ouvertes qui avaient comme objectif de laisser aux étudiants la possibilité de s'exprimer librement. Ces questions leur permettaient d'une part de décrire leurs représentations de la littérature et d'autre part de donner leur avis sur ce qui pourrait améliorer cet enseignement.

Les données que nous avons collectées ont été saisies sur des feuilles Excel afin de pouvoir être traitées. Notre analyse primaire a consisté à calculer l'importance, la satisfaction et l'influence de chacune de nos variables (Mucchielli, 1999: 55). L'utilisation du logiciel de statistique et d'économétrie STATA nous a permis d'exploiter nos données et surtout de faire le tri croisé des questions. Les tris à plat des réponses aux questions fermées uniques ont été représentés sous forme de camembert. Les graphiques présentés en barres ont été utilisés pour les questions à réponses multiples et les graphiques à colonnes pour représenter les moyennes des questions avec classement hiérarchique. Finalement, l'exploitation des réponses aux questions ouvertes a été faite à partir des morceaux choisis caractéristiques de chaque réponse. 
Les résultats de nos questionnaires nous ont permis de tirer quelques conclusions intéressantes concernant la nature, les méthodes et les objectifs assignés à l'enseignement de la littérature. Tout d'abord, les réponses des étudiants ont montré que leur niveau linguistique en français varie beaucoup et le plus souvent, se limite au niveau B2 (voir Graphiques 1 et 2).
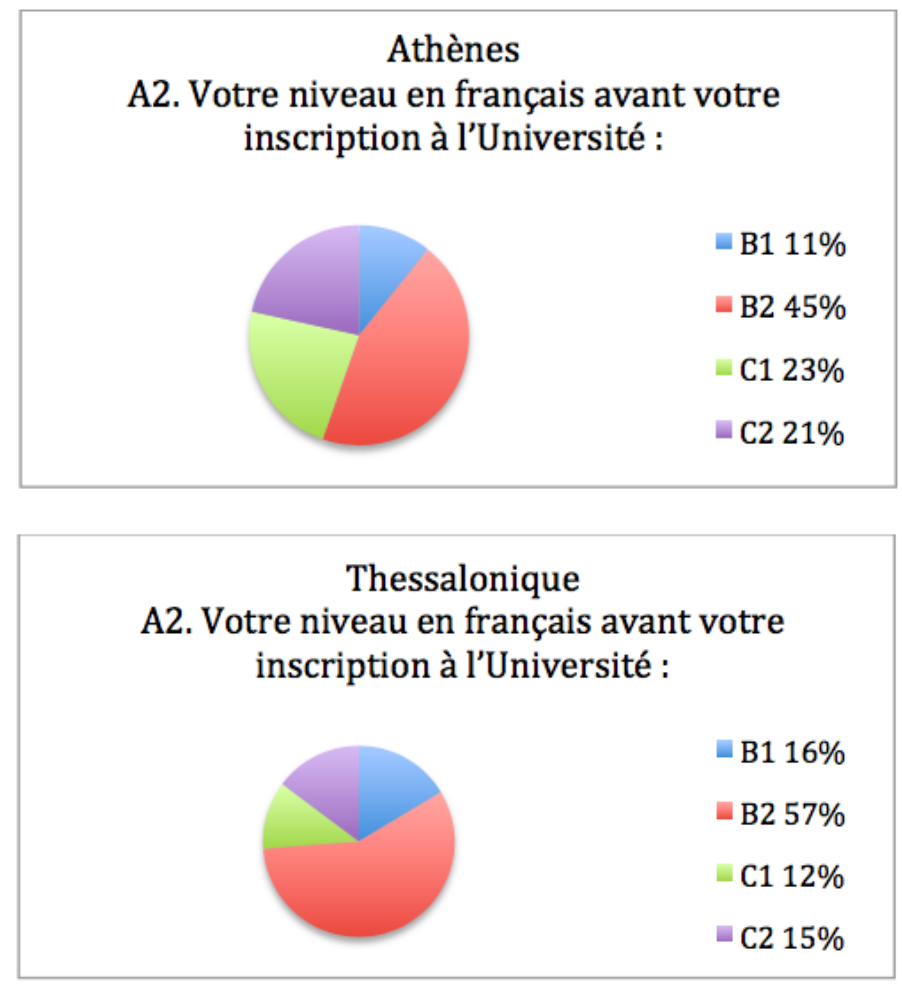

Graphiques 1 et 2 : Niveau linguistique des étudiants avant leur inscription à l'Université

En ce qui concerne les compétences développées lors des cours littéraires, nous avons constaté une différence significative entre les deux départements. Selon les étudiants d'Athènes, les cours littéraires leur permettent d'améliorer la langue. Dans ce cas, nous constatons que le texte littéraire est surtout utilisé pour des objectifs linguistiques. Le développement du vocabulaire vient ainsi en première place suivi de la créativité et de l'imagination. Les étudiants à Athènes déclarent aussi que les cours littéraires les aident à développer leur esprit critique. À l'Université de Thessalonique, la plupart des étudiants déclare que le texte littéraire leur permet de rencontrer la civilisation et la culture du pays. La créativité, l'imagination et le développement du vocabulaire viennent à la troisième et à la quatrième places. (Voir Graphiques 2 et 3 ). 

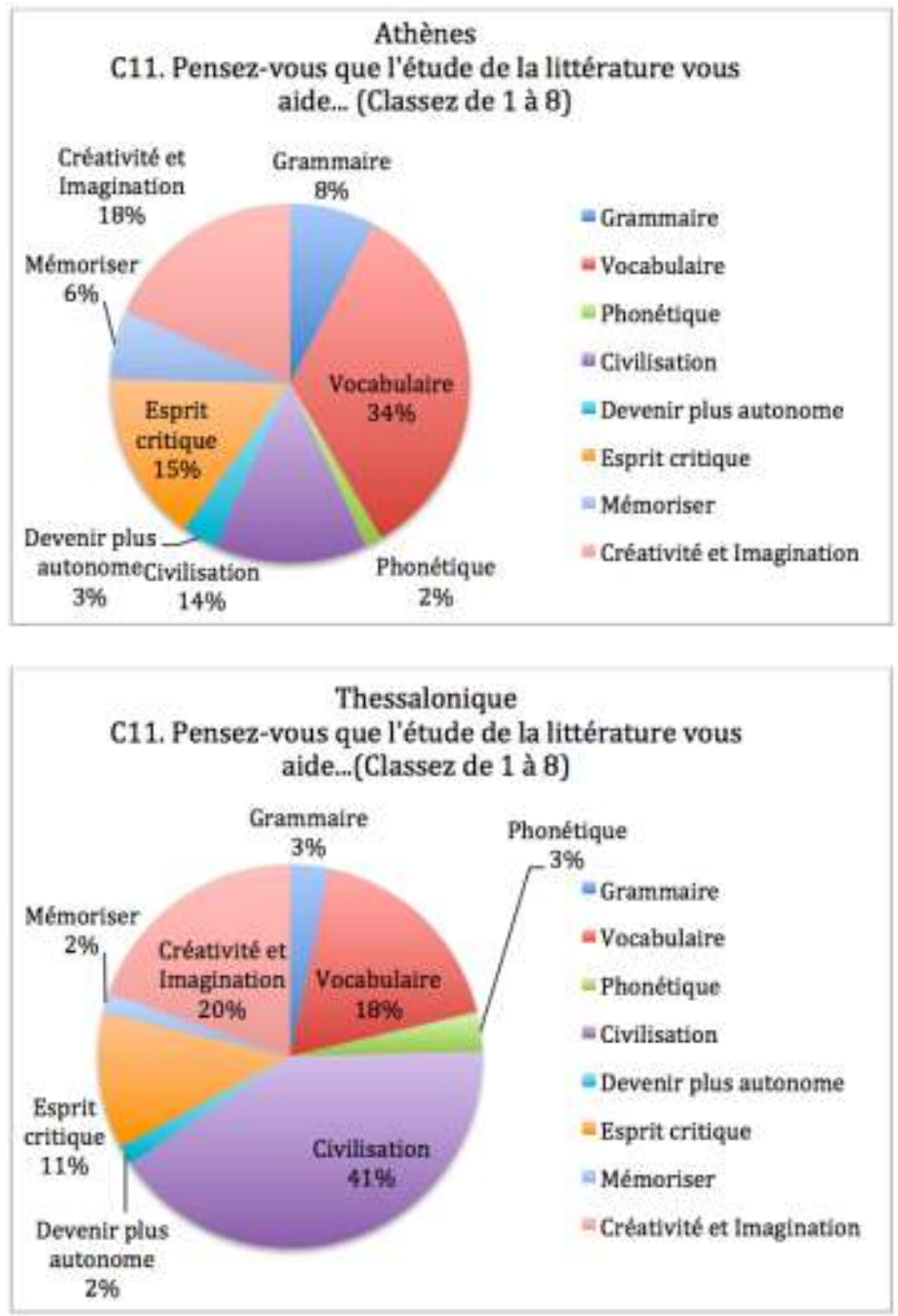

Graphiques 3 et 4 : Compétences développées lors des cours littéraires

Une échelle de Likert en 5 points a été utilisée afin de mesurer la fréquence (1 jamais - 5 toujours) de certaines pratiques en classe par les enseignants. Tout d'abord, nous constatons qu'il n'y pas de très grandes différences entre les réponses des étudiants des deux départements. En effet, la présentation de la biographie d'un auteur, comme méthode d'approche et de présentation d'un texte semble avoir une place prépondérante dans les cours littéraires. L'explication des mots inconnus, la lecture du passage par l'enseignant ainsi que la lecture critique sont les activités les plus fréquemment utilisées. Nous constatons par contre que les activités moins traditionnelles, comme l'écriture créative, la mise en scène théâtrale et l'utilisation de l'ordinateur occupent les dernières places. Les graphiques avec les moyennes cidessous représentent la fréquence des certaines activités aux deux départements. 

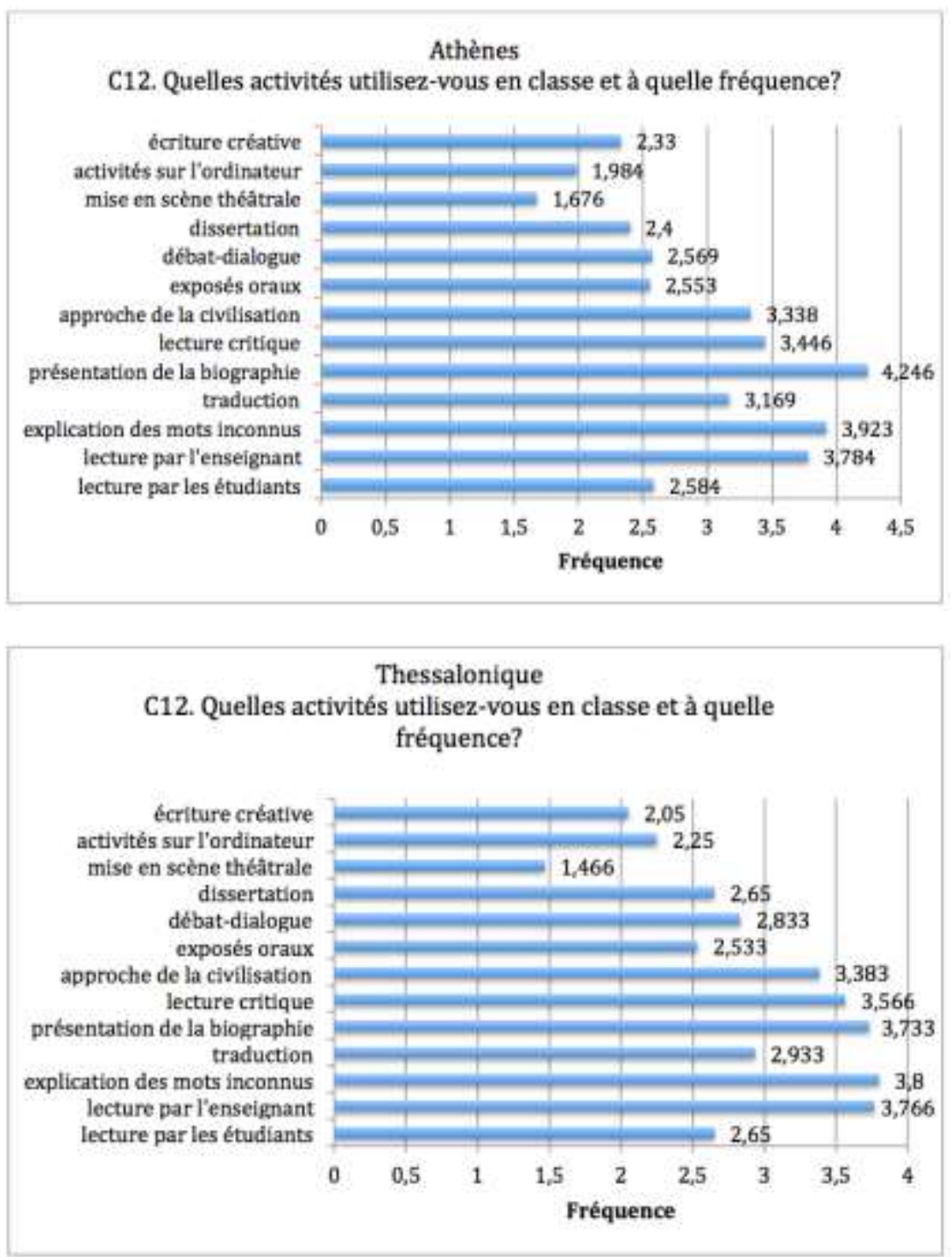

Graphiques 4 et 5 : Fréquence des activités utilisées en classe

Certaines questions nous ont permis d'appréhender les représentations et les images des étudiants sur l'objet de «littérature », telle qu'ils le conçoivent au sein de leur département. La plus grande majorité a déclaré que la littérature est l'expression d'une culture et qu'elle est associée à la civilisation et à l'histoire française. Concernant les auteurs les plus représentatifs, Victor Hugo, Charles Baudelaire et Honoré de Balzac apparaissent parmi les cinq premiers choix des étudiants. Inversement, les écrivains francophones ou d'expression française n'apparaissent qu'avec quelques citations disséminées. Nous constatons donc que l'enseignement de la littérature dans les deux départements grecs se restreint à la promotion d'une "culture cultivée » et son 
acquisition est perçue comme une «marque d'appartenance à la francité » (Porcher, $1995: 66)$.

\begin{tabular}{|l|l|r|}
\hline & Thessaloniki & $\begin{array}{r}\text { n.de } \\
\text { référe } \\
\text { nces }\end{array}$ \\
\hline 1. & HUGO & 37 \\
\hline 2. & BAUDELAIRE & 21 \\
\hline 3. & BALZAC & 20 \\
\hline 4. & MOLIERE & 20 \\
\hline 5. & CAMUS & 17 \\
\hline 6. & DE & 15 \\
\hline 7. & ZOLA & 15 \\
\hline
\end{tabular}

\begin{tabular}{|l|l|r|}
\hline & Athènes & $\begin{array}{r}\text { n. de } \\
\text { référe } \\
\text { nces }\end{array}$ \\
\hline 1. & HUGO & 33 \\
\hline 2. & BAUDELAIRE & 16 \\
\hline 3. & LAMARTINE & 14 \\
\hline 4. & ROUSSEAU & 14 \\
\hline $\mathbf{5 .}$ & BALZAC & 13 \\
\hline 6. & CAMUS & 12 \\
\hline 7. & CHATEAUBRIAND & 9 \\
\hline
\end{tabular}

Une des questions dont les résultats étaient intéressants pour notre recherche, proposait aux étudiants de classer de 1 à 6 les sections et les cours qu'ils préfèrent. Parmi les six sections (traduction, didactique, linguistique, littérature, civilisation, informatique), les étudiants des deux départements déclarent qu'ils préfèrent les cours de traduction. Il est possible que cette préférence soit due soit à la conviction que c'est une voie qui conduit au marché du travail, soit à la méthode d'enseignement de ces cours. La deuxième section de préférence pour les étudiants athéniens est la littérature. Par contre, à Thessaloniki les étudiants mettent en deuxième place les cours de didactique. Les camemberts ci-dessous illustrent le pourcentage de leur premier choix :

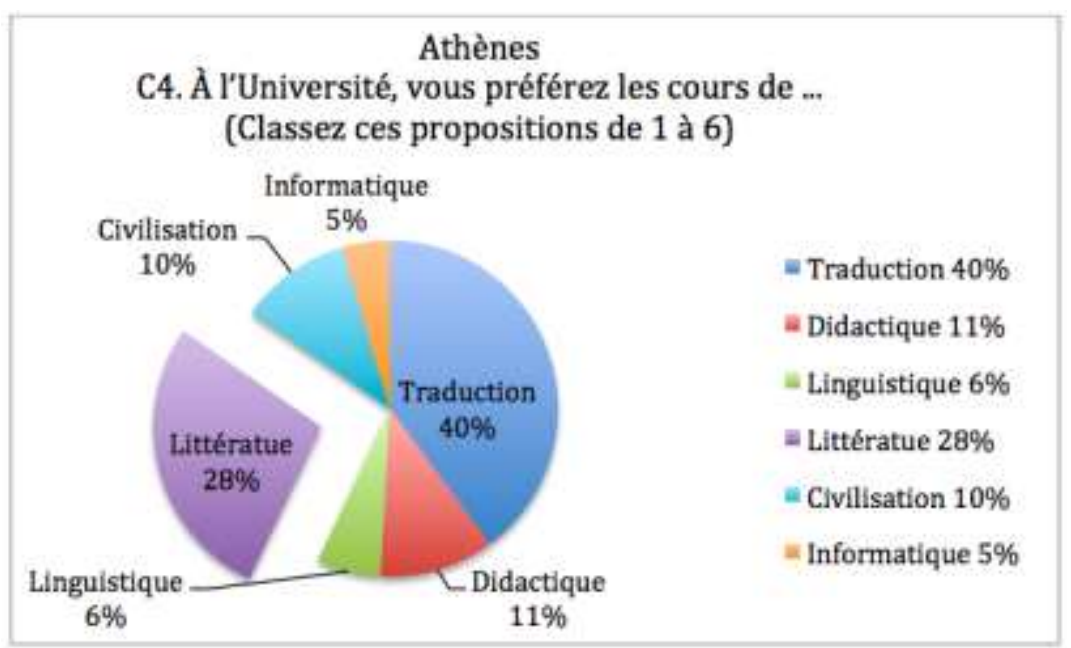




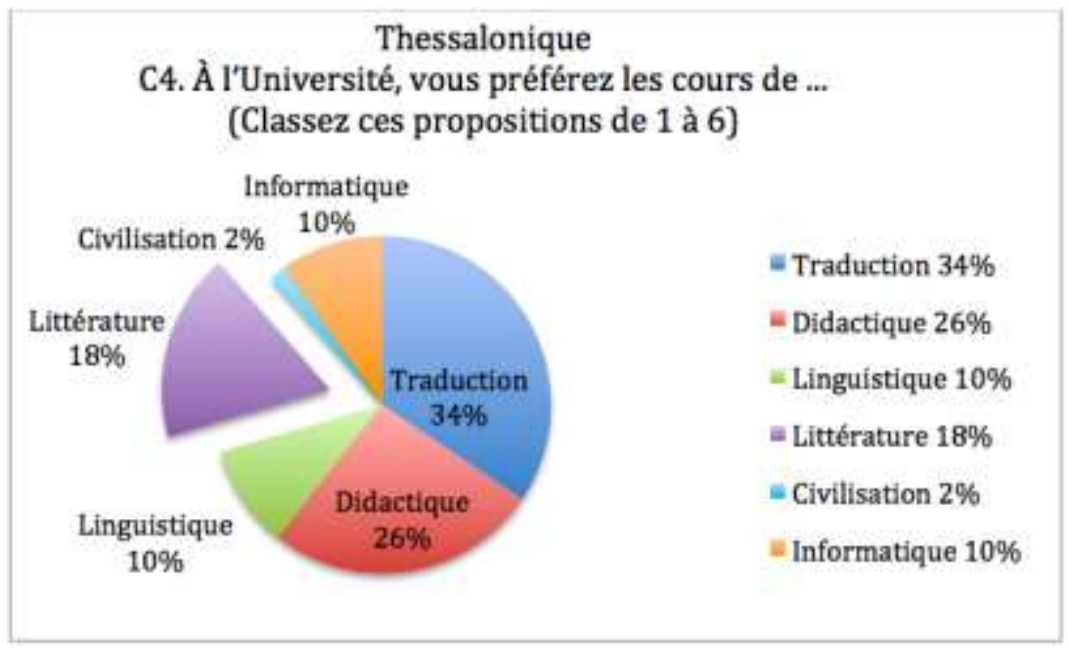

Graphiques 6 et 7 : Cours préférés par les étudiants

\section{L'enquête qualitative}

Après avoir obtenu une image globale des représentations et des pratiques au sein des deux départements, nous avons mené une étude qualitative qui nous a permis d'étudier en profondeur certains aspects. La première approche nous a permis de mesurer le niveau linguistique des étudiants, les auteurs les plus représentatifs, la fréquence de certaines approches d'enseignement ainsi que le degré de satisfaction des étudiants. La seconde a tenté de repérer les réactions, les expériences et les points de vue des étudiants et des enseignants face aux approches d'enseignement et face à l'objet de « littérature ».

Les 24 entretiens réalisés auprès des étudiants et les 9 entretiens menés auprès des enseignants nous ont donné une image assez représentative de la réalité. Notre guide d'entretien comprenait trois axes, correspondant aux trois thèmes principaux de notre étude : l'enseignement et l'apprentissage de la littérature, les représentations de la littérature, les évaluations et les propositions (voir le guide d'entretien en Annexe). Chacun de ces trois grands thèmes renvoyait à une série de questions et d'informations que nous souhaitions obtenir. Les deux guides d'entretien destinés aux étudiants et aux enseignants étaient très proches car nous avons voulu comprendre si leurs avis se rejoignaient ou non. Les étudiants ont spontanément parlé de l'apprentissage et les professeurs ont expliqué les méthodes d'enseignement. Les premiers ont décrit des séries d'actes et l'ensemble des activités dans lesquelles ils se sont engagés pour acquérir le savoir. Les seconds ont parlé des techniques, des méthodes et des moyens à mettre en œuvre pour transmettre le savoir.

Lorsque nous n'avons pas voulu que la langue soit un obstacle pour la fluidité du discours des étudiants, nous avons décidé de réaliser les entretiens en grec et, par la suite, nous les avons traduits en français. Ils ont été tous enregistrés, puis transcrits et insérés dans les annexes de notre thèse. Leur durée a varié entre 20 minutes et $1 \mathrm{~h} 40$. Chaque transcription est précédée d'un chapeau qui indique le contexte, c'est-à-dire le lieu, l'heure, la durée de l'entretien, le prénom de l'interviewé, son âge ainsi que l'année d'études où il est inscrit.

La traduction et les transcriptions des entretiens ont constitué notre matériel documentaire à partir duquel nous avons commencé à construire les données (Lessard-Hebert, Boutin, Goyette, 1997 : 70). Pour chacun des entretiens une fiche- 
résumé a été d'abord construite. L'analyse thématique et la préparation des grilles d'analyse catégorielle nous ont conduits à une lecture critique de nos données et nous ont permis de découper les transcriptions en unités, de les classer et de répertorier les thèmes pertinents et systématiques en lien avec nos hypothèses. Les données correspondant aux trois catégories ont été soulignées avec trois couleurs différentes afin d'être classifiés.

Les étudiants et les enseignants ont décrit les pratiques pédagogiques pour l'enseignement de la littérature. Parallèlement, ils ont partagé leurs images mentales construites, leurs perceptions ainsi que leurs représentations associées à l'objet de «littérature ». Enfin, les étudiants avaient l'occasion non seulement d'évaluer les enseignements littéraires mais aussi de proposer des pistes didactiques. La description des pratiques et des méthodologies a souvent été accompagnée de critiques de la part des étudiants.

Les enquêtés ont débuté l'entretien par une description générale de l'enseignement de la littérature dans leur département. Les descriptions des étudiants nous ont amené à comprendre les techniques et les pratiques utilisées dans les deux départements. A partir de l'ensemble des entretiens, nous avons repéré certaines caractéristiques traditionnelles de l'enseignement de la littérature comme les suivantes :

a. Les cours de type magistral

b. La traduction systématique des textes qui permet d'acquérir le nouveau vocabulaire

c. La place accordée à l'explication des structures grammaticales

d. La mémorisation, qui est au cœur des principes pédagogiques

e. L'utilisation de la série des manuels Lagarde et Michard jusqu'en 2010

f. La présentation de la biographie de l'auteur avant l'approche des textes, l'enseignement de la littérature sous l'influence de l'histoire littéraire, selon les préceptes de Gustave Lanson.

g. L'étude des textes de la littérature patrimoniale

En outre, les discours des enseignants et des étudiants ont révélé certaines représentations sur la littérature. D'abord, la littérature apparaît comme une des composantes prépondérantes de la culture française. Elle est directement liée à la culture, elle la caractérise et elle la définit. La littérature est aussi envisagée comme un voyage qui dépayse et qui permet la découverte de l'Autre et de l'ailleurs.

Ensuite, pour les étudiants grecs interrogés, la lecture et la littérature ont deux visages : d'un côté la lecture savante et légitime et de l'autre côté la lecture ordinaire qui procure plaisir et sensations. Selon les discours des étudiants, l'enseignement peut se définir comme un processus intimement lié à la transmission et l'obtention de savoirs, alors que la littérature serait une pratique, immédiatement liée à la jouissance esthétique et aux émotions qu'elle provoque. Les étudiants affirment que leurs lectures à l'extérieur de l'université ne s'accordent pas avec les textes enseignés. Ceci est dû au fait que les textes proposés par la norme éducative ne correspondent pas aux préférences des étudiants.

Les réponses des étudiants ont également soulevé un paradoxe. Tandis qu'ils affirment que la littérature est intéressante et indispensable pour leur culture, ils ne considèrent pas que son enseignement soit utile pour leur vie professionnelle, et par conséquent elle ne doit pas être enseignée. Les étudiants reconnaissent que la littérature contribue à élargir leurs horizons, à l'enrichissement de leurs savoirs, à la connaissance de nouvelles informations et à la rencontre avec la civilisation française. Pourtant, ils affirment qu'il est plus important d'être pourvu de savoirs et de compétences qui leur garantiront leur valorisation et évolution professionnelles. C'est pour cela qu'ils mettent l'accent sur les cours qu'ils considèrent susceptibles de les aider à s'insérer, et à répondre au mieux à l'environnement exigeant et compétitif du marché. 
Enfin, les étudiants avaient l'occasion non seulement d'évaluer les enseignements littéraires mais aussi de proposer des pistes didactiques. Ces propositions ont fait émerger les attentes et les intérêts des étudiants et nous ont donné la possibilité de réfléchir à des pistes didactiques susceptibles de renouveler les pratiques actuelles et d'alimenter les modes d'enseignement. Dans leur majorité, les étudiants ont manifesté leur désir de participer activement au cours et d'interagir avec leurs camarades. Les étudiants ont expliqué lors des entretiens que le cours magistral ne correspondait ni à leurs besoins ni à leurs attentes. En outre, ils ont affirmé que le recours aux TICE est capable d'influer de manière favorable sur leur motivation. Les débats en classe sur le sens caché des textes, l'étude des textes contemporains, la comparaison entre la littérature et le cinéma et entre la littérature et les autres formes artistiques, la mise en place des activités théâtrales sont des pratiques qui semblent intéressantes pour les étudiants.

\section{Conclusion}

Les résultats de deux enquêtes ont fait émerger les besoins et les intérêts des étudiants. Ils nous ont donné la possibilité de réfléchir à des pistes didactiques susceptibles de renouveler les pratiques actuelles et de contribuer à l'amélioration de l'enseignement de la littérature. La troisième et dernière partie de notre thèse s'est donc penchée sur l'élaboration des pistes didactiques adaptées au niveau et aux attentes du public.

Loin d'avoir épuisé la thématique de l'enseignement de la littérature, nous espérons que ce travail ouvre des perspectives de recherche concernant les innombrables terrains d'enquête potentiels et exprime finalement l'intérêt de la mise en place d'une recherche-action qui permettrait de tester les pistes pédagogiques élaborées in situ. Reproduire plusieurs expérimentations sur le terrain, réaliser des interventions régulières et systématiques, filmer les situations d'enseignement, distribuer des questionnaires d'évaluation aux étudiants nous permettraient d'assurer l'efficacité et la pertinence des dispositifs proposés ainsi que l'exactitude de nos conclusions.

Pour conclure, cette recherche a révélé les grands défis importants auxquels les études littéraires sont actuellement confrontées et qui obligent donc à les moderniser et à concevoir de nouvelles méthodes de transmission afin d'échapper aux reproches fréquents qui les définissent comme "trop érudites », «trop théoriques », «trop historiques » et «trop superficielles » (Baroni, Rodriguez, $2014: 13$ ) . Le sujet de la "crise des études littéraires" (Schaeffer, 2011: 110) et de la transmission de cet « héritage fragile » (Todorov, $2007: 90)$ est d'ailleurs identifié depuis longtemps et amène les chercheurs et les pédagogues à une remise en question constante des pratiques et des approches.

\section{Bibliographic references}

BARONI, R. - RODRIGUEZ, A. (dir.). 2014. Les passions en littérature. De la théorie à l'enseignement, Lausanne, Revue Études de Lettres, n. 295.

DENZIN, N. K. 1970. The research Act in Sociology, Chicago, Aldine.

HABHAB, S. 2006. Querelles autour des méthodes, In: La communication organisationnelle en question. Méthodes et méthodologies, Paris, L'Harmattan.

KARSENTI, T. 2006. Pragmatisme et Méthodologie de recherche en sciences de l'éducation: passons à la version 3.0, In: Formation et Profession, vol. 13, n. 1.

LESSARD-HEBERT, M. - BOUTIN, G. - GOYETTE, B. 1997. La recherche qualitative, fondements et pratiques, Bruxelles, De Boeck Université.

MUCCHIELLI, R. 1999. Le questionnaire dans l'enquête psycho-sociale, Paris, ESF, coll. « Formation Permanente en Sciences Humaines », $10^{\mathrm{e}}$ édition.

POISSON, Y. 1983. L'approche qualitative et l'approche quantitative dans les recherches en éducation, In: Revue des sciences de l'éducation, vol. 9, n. 3, pp. 379- 
368. Consulté le 25 novembre 2017, Available online: https://www.erudit.org/fr/revues/rse/1983-v9-n3-rse3487/900420ar/

PORCHER, L. 1995. Le français langue étrangère. Émergence et enseignement d'une discipline, Paris, Hachette.

SCHAEFFER, J.-M. 2011. Petite écologie des études littéraires. Pourquoi et comment étudier la littérature ?, Vincennes, Thierry Marchaisse.

TODOROV, T. 2007. La littérature en péril, Paris, Flammarion, coll. «Café Voltaire ».

\author{
Words 5604 \\ Characters 38011 (21,18 standard pages) \\ Prof. Assist. Angeliki Kordoni \\ Department of French as a foreign language \\ University Paris-Sorbonne Abu Dhabi \\ P.O. Box 38044, Abu Dhabi \\ United Arab Emirates \\ angeliki.kordoni@psuad.ac.ae
}


Annexe 1 - Questionnaire aux étudiants de langue et de littérature françaises

A. Identité

Prénom :

Age :...

Sexe : M / F

Langue(s) maternelle(s) :

a. Département d'Athènes b. Département de Thessaloniki

2. Votre niveau en français avant votre inscription à l'Université :

$\square$ Intermédiaire (B1) $\quad \square$ Avancé (C1)

Moyen (B2) $\quad \square$ Supérieur (C2)

Certificat(s) de langue française obtenu(s) avant votre inscription :

B. Pratiques de lecture

1. Avez-vous déjà suivi des cours de littérature française avant votre inscription à l'Université ?

Non Oui Si oui dans quel cadre?

2. Combien de livres avez-vous lu les 12 derniers mois? (lecture extra-scolaire, pour le plaisir)

1. plus de 10 livres

2. entre 5 et 10

3. entre 1 et 4

4. aucun

3. Dans quelles catégories classifierez-vous les livres que vous lisez ou que vous préférez lire ? (trois réponses au maximum)

1. Roman d'aventure

2. Roman Historique

3. Roman social

4. Roman érotique

5. Roman Policier

6. Roman de Science-fiction

7. Contes Philosophiques

8. Littérature de Voyage

9. Littérature de jeunesse

10. Roman politique

11. Théâtre

12. Poésie

C. Formation et situation actuelle

1. Vous êtes en : $\square 2^{\text {ème }}$ année d'études

$3^{\text {ème }}$

$4^{\text {ème }}$ ou plus

2. Selon vous, le programme actuel de votre département doit être :

a. réformé en profondeur

b. changé sur plusieurs points

c. amélioré sur quelques petits détails

d. conservé en l'état

3. Quels sont, selon vous, les changements les plus importants qu'il faut faire ?

4. À l'Université, vous préférez les cours ... (Classez ces propositions de 1 à 6)

$\square$ a. de traduction

$\square$ b. de didactique de FLE

$\square$ c. de linguistique

$\square$ d. de littérature

$\square$ e. de civilisation française

XLinguae, Volume 11 Issue 1XL, January 2018, ISSN 1337-8384, eISSN 2453-711X 
$\square$ f. d'informatique (logiciels de production d'activités pédagogiques)

5. Quels cours de littérature (obligatoires et optionnels) avez-vous déjà suivi ou suivez-vous pendant ce semestre?

- Moyen Age

- XVI ${ }^{\mathrm{e}}$ siècle

- XVII ${ }^{\mathrm{e}}$ siècle

- XVIII ${ }^{\mathrm{e}}$ siècle

- XIX ${ }^{\mathrm{e}}$ siècle

- Classicisme et Lumières

- Littérature Française II : le théâtre

$-\mathrm{XX}^{\mathrm{e}}$ siècle roman

- Analyse du texte littéraire I

- Introduction à la littérature européenne

- Dissertation littéraire (précisez aussi l'œuvre enseignée)

- Théorie du discours théâtral

- Littérature Française I : Poésie

- Littérature française III : le

- Analyse des textes littéraires et production de discours académique

- Nouvelles écritures poétiques et romanesques au $\mathrm{XX}^{\mathrm{e}}$ et au $\mathrm{XXI}^{\mathrm{e}}$ siècle

-Littérature francophone (Littérature en français écrite hors de France, p.ex. en Afrique, en Suisse, au Québec, en Belgique etc.)

-Autres, Précisez

6. Pensez-vous que les cours de littérature de votre Département sont :

a. Pas importants

b. peu importants

c. Importants

d. Très Importants

e. Extrêmement importants

7. En cours de littérature, aimeriez vous étudier une œuvre complète ou vous préférez les extraits ?

\section{Vous préférez étudier : (Classez de 1à 3)}
$\square$ a. le théâtre
$\square$ b. la poésie
$\square$ c. le roman (nouvelles, contes etc.)

9. Est-ce que vous avez déjà suivi un cours de littérature francophone? (Littérature belge, canadienne, littérature africaine, littérature francophone grecque etc.)

10. Connaissez-vous quelques auteurs qui écrivent en français et qui ne sont pas français?

11. Pensez-vous que l'étude de la littérature vous aide.... (Classez les avantages suivants dans l'ordre d'importance, du plus important au moins important, de 1 à 8)

a. à développer des compétences en grammaire

b. à enrichir le vocabulaire

c. à développer des compétences en phonétique

d. à rencontrer la culture et la civilisation françaises $\square$

e. à devenir plus autonome

f. à développer votre esprit critique

g. à mémoriser

h. à développer votre créativité et votre imagination

Autres, précisez

12. Quelles activités utilisez-vous en classe et à quelle fréquence ? 
13. Les textes littéraires étudiés correspondent-ils à vos goûts personnels ?

\begin{tabular}{|c|c|c|c|c|c|}
\hline Activités & $\begin{array}{l}\text { Ja- } \\
\text { mais }\end{array}$ & $\begin{array}{l}\text { Rare- } \\
\text { ment }\end{array}$ & $\begin{array}{l}\text { Quelque- } \\
\text { fois }\end{array}$ & Sou-vent & $\begin{array}{l}\text { Tou- } \\
\text { jours }\end{array}$ \\
\hline $\begin{array}{l}\text { lectures } \\
\text { oralisées de } \\
\text { passages en } \\
\text { classe par vous } \\
\text { /les étudiants }\end{array}$ & & & & & \\
\hline $\begin{array}{lr}\text { lectures } & \\
\text { oralisées } & \text { de } \\
\text { passages } & \text { en } \\
\text { classe } & \text { par } \\
\text { l'enseignant } & \end{array}$ & & & & & \\
\hline $\begin{array}{l}\text { explication des } \\
\text { mots inconnus }\end{array}$ & & & & & \\
\hline traduction & & & & & \\
\hline $\begin{array}{l}\text { présentation de } \\
\text { la biographie de } \\
\text { l'auteur }\end{array}$ & & & & & \\
\hline $\begin{array}{l}\text { lecture critique } \\
\text { des textes }\end{array}$ & & & & & \\
\hline $\begin{array}{l}\text { approche de la } \\
\text { civilisation }\end{array}$ & & & & & \\
\hline Exposés oraux & & & & & \\
\hline $\begin{array}{ll}\begin{array}{l}\text { débat } \\
\text { dialogue }\end{array} & \text { et } \\
\end{array}$ & & & & & \\
\hline dissertation & & & & & \\
\hline $\begin{array}{l}\text { mise en scène } \\
\text { théâtrale }\end{array}$ & & & & & \\
\hline $\begin{array}{ll}\text { activités } & \text { sur } \\
\text { l'ordinateur } & \end{array}$ & & & & & \\
\hline Ecriture créative & & & & & \\
\hline $\begin{array}{l}\text { Autres } \\
\text { (précisez) }\end{array}$ & & & & & \\
\hline
\end{tabular}

a. Jamais b. Rarement c. Quelquefois d. Souvent e. Toujours

14. L'étude des textes littéraires à l'Université vous donne-t-elle l'opportunité d'exprimer votre opinion personnelle et vos sentiments ?

a. Jamais b. Rarement c. Quelquefois d. Souvent e. Toujours

15. Aimeriez-vous utiliser les nouvelles technologies dans le cours de littérature ? a. Jamais b. Rarement c. Quelquefois d. Souvent e. Toujours

16. La création des ateliers d'écriture créative vous paraît-elle :

a. pas du tout intéressante $b$. pas intéressante c. moyennement intéressante $d$. intéressante

e. extrêmement intéressante

XLinguae, Volume 11 Issue 1XL, January 2018, ISSN 1337-8384, eISSN 2453-711X 
17. Selon vous, qu'est-ce qui pourrait rendre l'enseignement de la littérature plus attrayant et intéressant, selon vous ? (Quelles activités, quels textes, quels outils)

18. Que représente la littérature pour vous ? Décrivez en quelques mots

19. Pourquoi aimez-vous ou vous n'aimez pas lire?

20. Quels sont les 5 auteurs français ou francophones les plus importants à vos yeux ?

Je vous remercie d'avoir pris le temps de répondre au présent questionnaire. Nous tenons à préciser que les indications personnelles vous concernant ne seront en aucun cas cités. 


\section{SUJETS ET GRANDS AXES DES ENTRETIENS}

1.ENSEIGNEMENT ET APPRENTISSAGE DE LA LITTERATURE

Pourriez vous présenter le cours de littérature dans votre département ?

- Quels cours littéraires avez-vous déjà suivi?

- Quels cours littéraires enseignez-vous ou avez-vous enseigné dans le passé? (question pour les profs)

- Quels manuels vous utilisez?

- Quels textes vous étudiez?

- Combien d'heures par semaine?

- Travaillez-vous des extraits ou des cuvres complètes?

- Quels sont les plus grandes difficultés d'un texte littéraire?

- Quelles activités de classe vous préférez/ utilisez?

- Quelles sont les activités que vous n'appréciez pas?

- Y a t-il-une évolution méthodologique pendant les dernières années/ décennies? (p. ex. diversification des corpus -question pour les profs)

- Quelles finalités et objectifs assignez-vous à cet enseignement? (question pour les profs)

\section{REPRÉSENTATIONS SUR LA LITTÉRATURE}

D'après vous, l'étude de la littérature est utile à l'apprentissage d'une langue étrangère ?

- Quels sont selon vous les avantages les plus importants de l'étude du texte littéraire?

- Pourquoi étudier la littérature française dans le département de FLE? (question pour les profs)

- Vous trouvez qu'il y a d'autres séminaires plus utiles de la littérature? Pourquoi?

- Qu'est-ce que la littérature représente pour vous? 


\section{EVALUER ET PROPOSER}

Qu'est-ce qu'on pourrait faire pour rendre l'enseignement littéraire plus vivant et attrayant?

- Vous êtes satisfait de l'enseignement de la littérature dans votre département?

- Quels sont, selon vous, les problèmes principaux de cet enseignement littéraire?

- Si le cours de littérature était un cours optionnel, le choisiriez-vous ?

- D’après vous, l'utilisation des nouvelles technologies dans la classe de littérature est possible? Comment?

- Comment pourrait-on améliorer l'enseignement littéraire?

- Quelles autres activités vous croyez qu'elles seraient intéressantes et motivantes pour les étudiants?

- Voulez-vous rajouter quelque chose sur ce sujet? 